\title{
Artéria lienal em coelhos Nova Zelândia: estudo anatômico destinado a pesquisa experimental e prática cirúrgica
}

\section{Lienal artery in New Zealand rabbit: Anatomical study for experimental research and surgical practice}

\author{
Bárbara Xavier Silva'; Magno Santos Roza²; Ilma Cely Amorim Ribeiro ${ }^{3}$; Márcio \\ Antonio Babinski ${ }^{4}$; Marcelo Abidu Figueiredo ${ }^{5 *}$
}

Resumo

\begin{abstract}
Na presente pesquisa foram utilizados 30 espécimes de coelhos adultos Nova Zelândia, 13 machos e 17 fêmeas. Após fixação com solução de formaldeido a $10 \%$ e preenchimento do sistema arterial com solução de látex corado procedeu-se a dissecção para a observação da disposição das artérias lienais. A análise estatística foi realizada através teste do $X^{2}$ (Qui-quadrado), ao nível de significância de 5\%, para testar se a hipótese de nulidade é verdadeira para o número de ramificações principais da artéria lienal. As relações do comprimento da artéria lienal e do baço em função do comprimento rostro-sacral foram calculadas através do coeficiente de correlação " $r$ ", que pode variar entre -1 e +1 , isto é, $-1 \leq$ $\mathrm{r} \leq+1$. A média do comprimento da artéria lienal foi de 2,166 cm. Em todos os coelhos dissecados $(100 \%)$ a artéria lienal se originou diretamente da artéria celíaca como uma artéria única, emitindo diferentes ramos para o baço: um ramo em sete $(23,3 \%)$ animais, dois ramos em sete $(23,3 \%)$ animais, três ramos em $10(33,3 \%)$ animais, quatro ramos em quatro $(13,3 \%)$ animais e cinco ramos em dois $(6,7 \%)$ animais. Do baço a artéria lienal emitiu diferentes números de ramos para a grande curvatura do estomago: um ramo em sete $(23,3 \%)$ animais, dois em quatro $(13,3 \%)$ animais, três em cinco $(16,7 \%)$ animais, quatro em três $(10 \%)$, e cinco em apenas um $(3,3 \%)$ animal. Foi observada correlação positiva entre o comprimento do baço e da artéria lienal em função do comprimento rostro-sacral. O número de ramificações da artéria lienal independe do sexo do animal.
\end{abstract}

Palavras-chave: Baço. Coelho. Vascularização.

\begin{abstract}
In the study 30 specimens of adult New Zealand rabbits, 13 males and 17 females were used. After fixed with $10 \%$ formaldehyde solution and performing the injections with latex solution stained with pigment, the animals were dissected to identify the disposition of the lienal arteries. The statistical analysis was made by the Qui-square $\left(\mathrm{X}^{2}\right)$ test, with a $5 \%$ level of significance, to test if the nullity hypothesis is true for the of lienal artery main ramifications. The relations between the lienal artery and spleen length with rostrum-sacral length were calculated by the correlation coefficient " $r$ " varying between -1 and +1 , that is, $-1 \leq \mathrm{r} \leq+1$. The mean length of the lienal artery was $2,16 \mathrm{~cm}$. In all $(100 \%)$
\end{abstract}

\footnotetext{
1 Médica Veterinária Autônoma, Mestre em Medicina Veterinária, Patologia e Ciências Clínicas, UFRRJ. E-mail: babi.xavier@ gmail.com

2 Discente de Graduação em Zootecnia, Monitor da Área de Anatomia Animal, UFRRJ, Seropédica, RJ. E-mail: magno2sr@ hotmail.com

3 Médica Veterinária Autônoma e Discente de Pós-Graduação em Medicina Veterinária, UFF. E-mail: ilmavetuff@gmail.com

4 Prof. Adjunto do Departamento de Morfologia, UFF, Niterói, RJ. E-mail: mababinski@gmail.com

5 Médico Veterinário, Prof. Adjunto do Departamento de Biologia Animal, Área de Anatomia Animal, UFRRJ, Seropédica, RJ. E-mail: marceloabidu@gmail.com

* Autor para correspondência
} 
dissected rabbits the lienal artery, originated directly from the celiac artery as an only one artery giving different numbers of branches to the spleen: one branch in seven $(23.3 \%)$ animals, two branches in seven $(23.3 \%)$ animals, three branches in $10(33.3 \%)$ animals, four branches in four $(13.3 \%)$ animals and five branches in two (6.7\%) animals. From the spleen, the lienal artery gave different numbers of branches to the stomach greater curvature: one branch in seven $(23.3 \%)$ animals, two in four $(13.3 \%)$ animals, three in five (16.7\%) animals, four in three (10\%), and five in only one (3.3\%) animal. Positive correlation was observed between the lienal artery and spleen length with the rostrum-sacral length. The number of lienal artery branches is not gender dependent.

Key words: Spleen. Rabbit. Vascularization.

\section{Introdução}

O baço desempenha função relevante sobre a imunidade e os elementos figurados do sangue, tanto na série vermelha como na branca. Protege o organismo nas queimaduras térmicas por meio do fator esplênico lipoidal e diminui a predisposição à embolia microvascular pulmonar (ESPOSITO et al., 2003; SOUZA et al., 2005).

A vascularização arterial do baço em mamíferos é feita pela artéria lienal, que pode se originar diretamente da artéria celíaca ou através de um tronco comum formado pelas artérias gástrica esquerda e lienal, denominado de tronco gastro-lienal (NICKEL; SCHUMMER; SEIFERLE, 1983; ABIDUFIGUEIREDO et al., 2005, 2008; XAVIER-SILVA et al., 2011).

A esplenectomia foi muito difundida e obteve grande aceitação, principalmente em relação ao trauma do baço, pela facilidade técnica e por coibir efetivamente a hemorragia.

Durante mais da metade do século XX, a esplenectomia foi a principal indicação em caso de trauma de baço. Acreditava-se que a sua função não era importante e que a asplenia não causava nenhuma conseqüência no longo prazo (PISTER; PACHTER, 1994; ZÚÑIGA et al., 2002). A partir de 1919 começou a se perceber que a esplenectomia aumentava a susceptibilidade a infecções (KRIVIT; GIEBINK; LEONARD, 1979).

Esse fato alcançou repercussão quando King e Shumacker Junior (1952) relataram a ocorrência de infecção fulminante pós-esplenectomia. Seguiram-se relatos clínicos e trabalhos experimentais de infecção grave pós esplenectomia que demonstravam maior susceptibilidade de animais asplênicos à infecção (RODRIGUES et al., 1991; SOUZA et al., 2005).

Observações clínicas e experimentais têm demonstrado que, após a esplenectomia, ocorrem alterações na imunidade, representadas pela diminuição da IgM sérica, redução da capacidade de opsonização do soro e incapacidade e cessação da produção de tuftisina (tetrapeptídeo facilitador da fagocitose bacteriana) (NUNES et al., 1994; BRANDT et al., 2001; PETROIANU, 2001a).

A infecção grave após a esplenectomia não é uma contra indicação absoluta. A indicação necessita ser precisa e bem fundamentada e com benefícios que superem os riscos (HEIL et al., 2001; SOUZA et al., 2005).

O melhor conhecimento das conseqüências da esplenectomia acarretou mudanças na conduta cirúrgica em relação ao tecido esplênico voltada à sua conservação, como esplenectomias parciais, tamponamentos, aplicação tópica de hemostáticos e embolização (ESPOSITO et al., 2003; RESENDE; PETROIANU, 2002; ZÚÑIGA et al., 2002).

Nas situações em que a esplenectomia é inevitável, como em alguns tipos de trauma e doenças hematológicas, é recomendado preservar baços acessórios e realizar o implante de tecido esplênico (RODRIGUES et al., 1991; PISTER; PACHTER, 1994; ESPOSITO et al., 2003; BRANDT et al., 2001; PETROIANU, 2001b; MARQUES et al., 2002; SOUZA et al., 2005; SIMÕES et al., 2007).

O objetivo deste artigo foi descrever a vascularização arterial do baço de coelhos, fornecendo dessa forma subsídios para a área básica e cirurgia experimental. 


\section{Material e Métodos}

Os estudos de dissecção anatômica foram realizados em 30 coelhos, 13 machos e 17 fềmeas, pesando em media $2,5 \mathrm{~kg}$ e com média de comprimento rostro-sacral de $40,89 \mathrm{~cm}$. Todos os animais utilizados faziam parte das aulas práticas das disciplinas de Cirurgia e foram cedidos à área de Anatomia Animal, já submetidos à eutanásia. Esta publicação faz parte do projeto intitulado Anatomia Topográfica e Sistêmica do Coelho: estudo destinado a pratica cirúrgica, radiológica e experimental, que foi submetido e aprovado pelo Comitê de Ética e Pesquisa da Universidade Federal Rural do Rio de Janeiro (Processo número 23083.002379/2007-08).

Os coelhos foram posicionados em decúbito lateral direito e em seguida o tórax foi aberto e dissecado para evidenciação da porção torácica da artéria aorta, que foi canulada. Desse modo, o sistema arterial foi "lavado" com solução fisiológica de $\mathrm{NaCla} 0,9 \%$, sendo realizado a seguir a fixação com solução de formaldeído a $10 \%$ conforme técnica anatômica padrão. Em seguida os vasos foram preenchidos com solução de petrolátex S65 corado com pigmento vermelho. Os animais permaneceram acondicionados em cubas com solução de formaldeído a $10 \%$ por 5 dias para polimerização do látex. Os vasos lienais foram dissecados, fotografados para documentação e denominados de acordo com a Nomenclatura Anatômica Veterinária (SCHALLER, 1999). Com auxilio de um paquímetro de precisão o baço e a artéria lienal foram mensurados. Também foram feitos desenhos esquemáticos.

\section{Análise estatística}

Com o intuito de verificar se a distribuição de freqüências observadas para os 30 animais examinados está de acordo com a teoria, aplicou-se o teste do $\mathrm{X}^{2}$ (Qui-quadrado), ao nível de significância de 5\%, para testar se a hipótese de nulidade é verdadeira, isto é, se o número de ramificações da artéria lienal não depende do sexo do animal. As relações do comprimento da artéria lienal em função do comprimento rostro-sacral e o comprimento do baço em função do comprimento rostro-sacral foram calculadas através do coeficiente de correlação "r", que pode variar entre $-1 \mathrm{e}+1$, isto é, $-1 \leq$ $\mathrm{r} \leq+1$. Considerou-se um $\mathrm{p}<0,05$ como significativo. Todo o processamento dos dados foi realizado no Software Grafpad Instat 5.

\section{Resultados}

A média do comprimento da artéria lienal foi de 2,16 $\mathrm{cm} \pm 0,14$ e o baço apresentou média de comprimento de 4,34 $\mathrm{cm} \pm 0,28$ (Figura 1). Em todos os coelhos dissecados (100\%) a artéria lienal se originou diretamente da artéria celíaca como uma artéria única (Figuras 2, 3). Emitiu dois ramos pancreáticos em 10 animais (33,33\%) e quatro ramos em 20 animais (66,7\%); em seguida um ramo gástrico em 13 animais $(43,33 \%)$ e dois ramos em 17 animais (56,7\%); e apenas um ramo gastrolienal antes do hilo esplênico em 16 animais $(53,33 \%)$.

Ao penetrar no ligamento gastro-lienal a artéria lienal emitiu dois ramos pancreáticos em 20 animais $(66,7 \%)$ e quatro ramos em 10 animais (33,33\%), e diferentes ramos para o baço: um ramo em sete animais $(23,3 \%)$, dois ramos em sete animais $(23,3 \%)$, três ramos em 10 animais $(33,3 \%)$, quatro ramos em quatro animais $(13,3 \%)$ e cinco ramos em dois animais $(6,7 \%)$. Do baço a artéria lienal emitiu diferentes números de ramos para a grande curvatura do estomago (artérias gástricas curtas): um ramo em sete animais (23,3\%), dois em quatro animais (13,3\%), três em cinco animais $(16,7 \%)$, quatro em três animais $(10 \%)$, e cinco em apenas um animal (3,3\%). Após contornar a face visceral do baço a artéria lienal terminou bifurcando-se em uma artéria gastro-epiplóica esquerda e uma artéria omental.

Foi observada correlação positiva $(r=0,4795$, $\mathrm{p}=0,0073$ ) entre o comprimento da artéria lienal e o comprimento rostro-sacral e entre o comprimento do baço e o comprimento rostro-sacral $(\mathrm{r}=0,9315, \mathrm{p}=$ 0,0001).

$(\mathrm{r}=0,91)$. O numero de ramificações da artéria lienal independe do sexo do animal. 


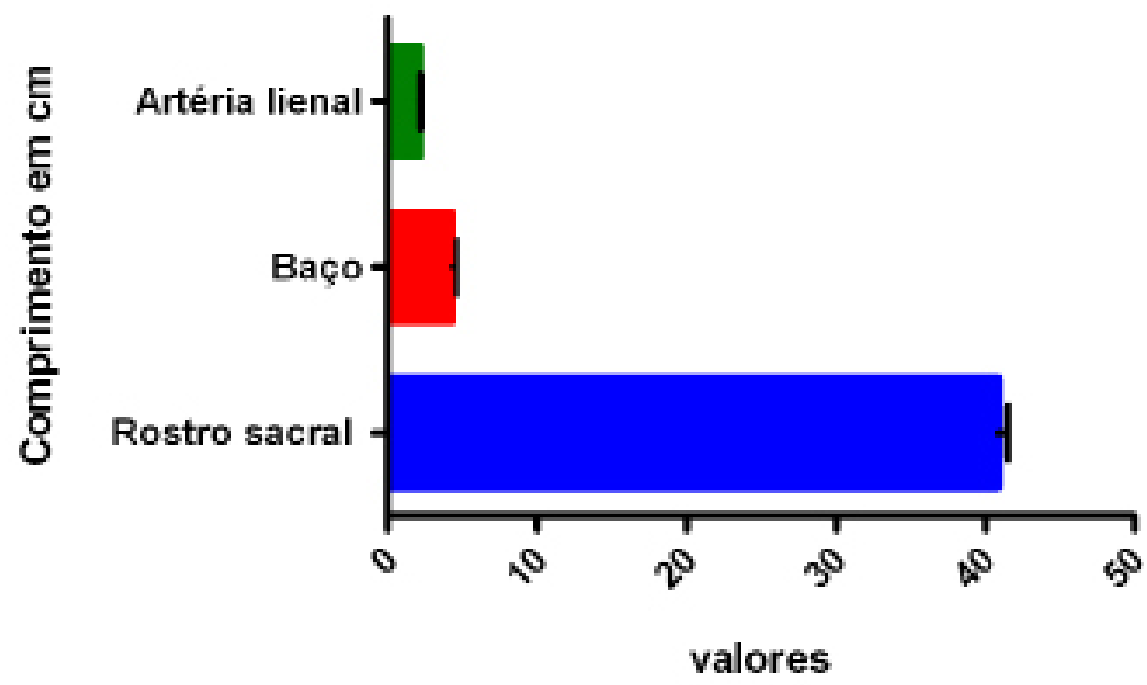

Figura 1. Média e desvio padrão do comprimento rostro-sacral, do baço e da artéria lienal de coelhos Nova Zelândia.

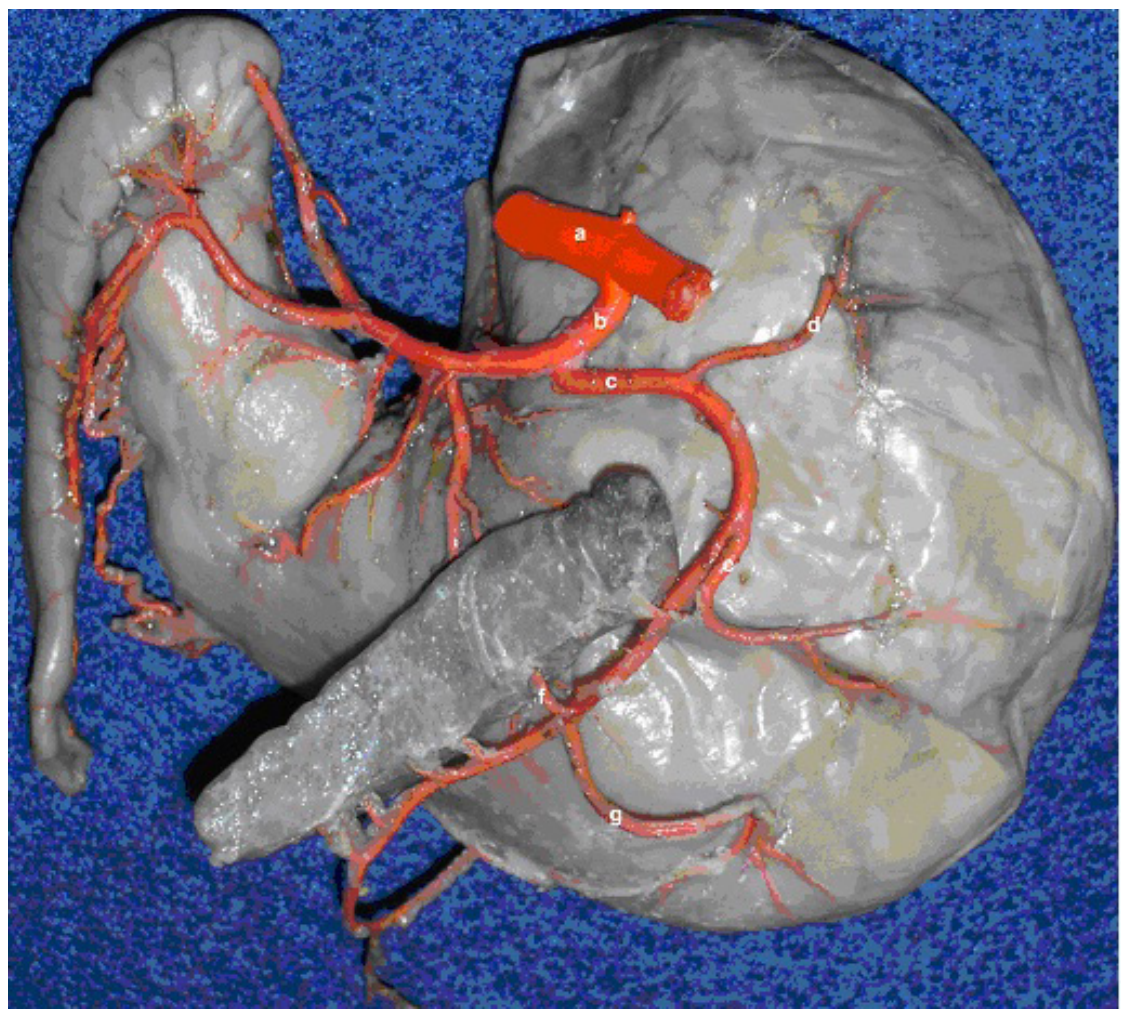

Figura 2. Fotomacrografia da face visceral do estômago demonstrando a artéria lienal e suas principais ramificações: (a) Aorta, (b) Artéria celíaca, (c) Artéria lienal, (d) ramo gástrico, (e) ramo gastro-lienal, (f) ramo lienal, (g) Artéria 


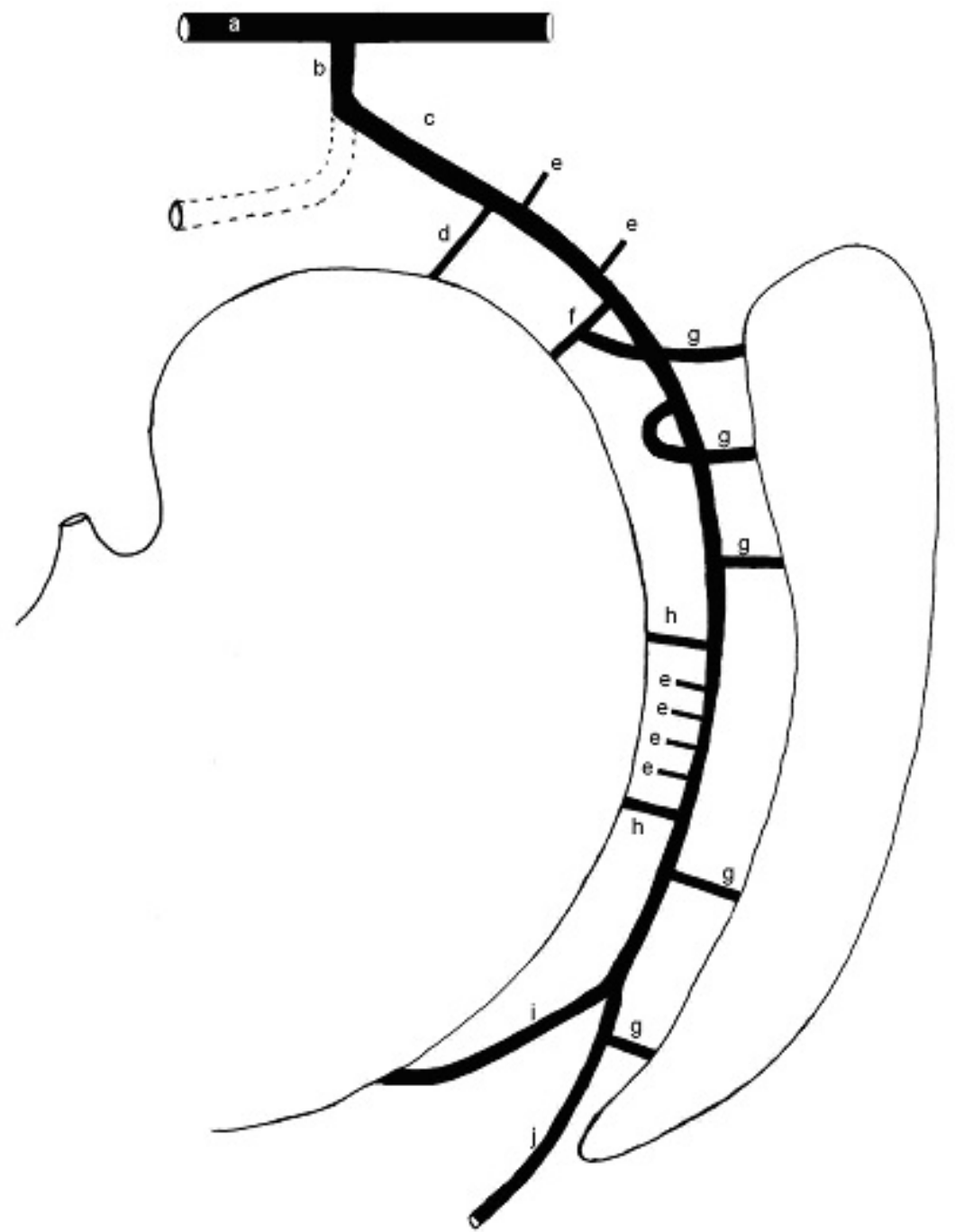

Figura 3. Desenho esquemático demonstrando a artéria lienal e suas principais ramificações: (a) Aorta, (b) Artéria celíaca, (c) Artéria lienal, (d) ramo gástrico, (e) ramo pancreático, (f) ramo gastro-lienal, (g) ramo lienal, (h) Artéria gástrica curta, (i) Artéria gástro-epiplóica esquerda, (j) Artéria omental.

\section{Discussão}

De acordo com Nickel, Schummer e Seiferle (1983), a artéria lienal se origina diretamente da artéria celíaca em todos os animais domésticos. Entretanto, Schmidt, Lohse e Suter (1980) relataram uma rara variação onde a artéria lienal se originou diretamente da artéria mesentérica cranial em cão, variação esta não encontrada nesta pesquisa.

Ainda em cães, a artéria lienal pode apresentar sua origem diretamente da artéria celíaca onde se verifica a emergência separada da artéria hepática, gástrica esquerda e lienal, a trifurcação clássica, ou através de arranjo onde ocorre a emergência separada da artéria hepática seguida por um tronco comum formado pela artéria gástrica esquerda e a artéria lienal, denominado de tronco gastro-lienal em 33,3\% e $56,7 \%$, respectivamente (ABIDU-FIGUEIREDO et al., 2005).Na presente pesquisa a artéria lienal se originou diretamente da artéria celíaca como uma artéria única em todos os animais dissecados.

De acordo com Xavier-Silva et al. (2011), no gato artéria lienal emerge como um vaso ímpar diretamente da artéria celíaca isoladamente ou através da formação de um tronco comum com a artéria gástrica esquerda. Em ambos os casos deu 
origem a ramos para o lobo esquerdo do pâncreas, $\mathrm{o}$ estômago e o baço. A artéria lienal em fềmeas emerge diretamente da artéria celíaca através da trifurcação clássica formada pelas artérias hepática, gástrica esquerda e lienal em $46,7 \%$ dos animais e em $53,3 \%$ a origem se deu pela formação de um tronco comum com a artéria gástrica esquerda (tronco gastro-lienal). Nos machos a artéria lienal emerge diretamente da artéria celíaca através da trifurcação clássica em $53,3 \%$ dos animais e em $46,7 \%$ a origem se deu pela formação de um tronco comum com a artéria gástrica esquerda.

Nos coelhos deste estudo a artéria lienal se originou diretamente da artéria celíaca como uma artéria impar em 100\% dos animais dissecados, resultado também demonstrado em eqüinos, onde a artéria lienal se origina diretamente da artéria celíaca que é formada pelas artérias hepática, lienal e gástrica esquerda (GETTY, 1986; NICKEL; SCHUMMER; SEIFERLE, 1979, 1983).

Nos ruminantes a artéria lienal se origina diretamente da artéria celíaca que é formada pelas artérias hepática, lienal e gástrica esquerda (LANGENFELD; PASTEA, 1977; GETTY, 1986; NICKEL; SCHUMMER; SEIFERLE, 1979, 1983), corroborando com os resultados obtidos na presente pesquisa.

Gonçalez et al. (2003) estudaram a origem e as ramificações da artéria celíaca em fetos suínos e observaram que em $96,6 \%$ dos casos a artéria lienal se originou diretamente da artéria celíaca que apresentava como ramos principais as artérias hepática, lienal e gástrica esquerda; em apenas um animal a artéria celíaca estava ausente e as artérias lienal e hepática tiveram sua origem diretamente da aorta.

No homem Bergman et al. (1988) relataram a origem da artéria lienal à partir dos seguintes arranjos: uma quadrifurcação formada por duas artéria gástricas, artéria lienal e artéria hepática em $7 \%$ dos casos estudados; tronco hepato-lienal em 5\% e tronco gastro-lieinal em 3\%, discordando dos dados obtidos na presente investigação onde a origem foi diretamente da artéria celíaca sem formação de troncos em todos os animais dissecados.

O arranjo da artéria lienal em coelhos e suas variações apresentam relevância nos estudos experimentais, pois a esplenectomia total vem decrescendo e relatos clínicos e trabalhos experimentais demonstraram infecção grave pós esplenectomia com maior susceptibilidade de animais asplênicos à infecção (RODRIGUES et al., 1991; SOUZA et al., 2005).

Através do conhecimento preciso das variações da artéria lienal em coelhos é possível determinar as indicações e conseqüências da esplenectomia em relação à conservação do tecido esplênico como nas esplenectomias parciais, tamponamentos, aplicação tópica de hemostáticos, embolização e auto implante esplênico (ESPOSITO et al., 2003; PETROIANU, 2001b; MARQUES et al., 2002; RESENDE; PETROIANU, 2002; ZÚÑIGA et al., 2002; SOUZA et al., 2005; SIMÕES et al., 2007).

Além disso, os resultados obtidos na presente investigação fornecem subsídios importantes para a realização e interpretação de delineamentos ao usar o coelho como modelo na prática cirúrgica, radiológica e experimental.

\section{Referências}

ABIDU FIGUEIREDO, M.; DIAS, G. P.; CERUTTI, S.; CARVALHO DE SOUZA, B.; MAIA, R. S; BABINSKI, M. A. Variations of celiac artery in dogs: anatomic study for experimental, surgical and radiological practice. Int. $J$. Morphol, Chile, v. 23, n. 1 p. 37-42, 2005.

ABIDU FIGUEIREDO, M.; XAVIER SILVA, B.; CARDINOT, T. M.; BABINSKI, M. A.; CHAGAS, M. A. Celiac artery in New Zealand rabbit: anatomical study of its origin and arrangement for experimental research and surgical practice. Pesq. Vet. Bras., Rio de Janeiro, v. 28, n. 5, p. 237-240, 2008.

BERGMAN, R. A.; THOMPSON, S. A.; AFIFI, A. K.; SAADEH, F. A. Compendium of human anatomic variation: catalog, atlas and world literature. Baltimore: Urban and Schwarzenberg, 1988. 593 p. 
BRANDT, C. T.; MACIEL, D. T.; FREI CANECA, A. O.; CASTRO, C. M. B.; ARAÚJO, L. B. Autotransplant of spleen tissue in children with schistosomiasis: Evaluation of splenic function after splenosis. Mem. Inst. Oswaldo Cruz, Rio de Janeiro, v . 96, p. 117-122, 2001. Suplemento.

ESPOSITO, T. J.; GAMELLI, R. L. Injury to the spleen. In: MATTOX, K. R.; FELICIANO, D. U.; MOORE E, E. (Ed.). Trauma. 4th ed. New York : McGraw-Hill Medical Publishing Division, 2003. p. 683-710.

GETTY, R. Anatomia dos animais domésticos. 5. ed. Rio de Janeiro: Guanabara Koogan, 1986. 2000 p.

GONÇALEZ, P. O.; CARNEIRO E SILVA, F. O.; SEVERINO, R. S.; DRUMMOND, S. S. Origens e ramificações da artéria celíaca em fetos de suínos (Sus scrofa domesticus Linnaeus, 1758) da linhagem "Rezende". Biosci. J., Uberlândia, v. 19, n. 1, p. 87-95, 2003.

HEIL, R. I.; MALAFAIA, O.; MATIAS, J. E. F.; RIBAS FILHO, J. M.; CAMPOS, A. C. L.; MELLO, L. R.; SIMÕES, J. C. Viabilidade histológica do autotransplante esplênico em omento maior, retroperitôneo, mesentério e tecido subcutâneo de cães submetidos à esplenectomia total. Arq. Bras. Cir. Dig., São Paulo, v. 14, n. 4, p. 174-77, 2001.

KING, H.; SHUMACKER JUNIOR, H. B. Splenic studies. I. Susceptibility to infection after splenectomy performed in infancy. Ann Surg., Madison, v. 135, n. 2, p. 239-242, 1952.

KRIVIT, W.; GIEBINK, G. S.; LEONARD, A. Overwhelming postsplenectomy. Surg. Clin. North Am., Pennsylvania, v. 9, n. 2, p. 223-233, 1979.

LANGENFELD, M.; PASTEA, E. Anatomical variants of the celiac artery in sheep with special reference to the celiac mesenteric arterial trunk. Anat. Anz., Jena, v. 142, n. 3, p. 168-174, 1977.

MARQUES, R. G.; PETROIANU, A.; COELHO, J. M.; PORTELA, M. C. Regeneration of splenic autotransplants. Ann. Hematol., Hannover, v. 8, n. 6, p. 622-626, 2002.

NICKEL, R.; SCHUMMER, A.; SEIFERLE, E. The viscera of domestic mammals. $2^{\text {nd }}$ ed. Berlin: Verlag Paul Parey, 1979. 540 p.

The anatomy of the domestic animals. 2nd ed. Berlin: Verlag Paul Parey, 1983. 610 p.

NUNES, C. C. A.; NUNES, W. V.; WAECHTER, F. L.; PEREIRA LIMA, L. Trauma esplênico: valor do autotransplante. Revta Med. Sta Casa, Porto Alegre, v. 5, n. 10, p. 1038-1044, 1994.

PETROIANU, A. Cirurgia conservadora do baço
In: (Ed.). Terapêutica cirúrgica: indicações, decisões, tática, técnica. Rio de Janeiro: Guanabara Koogan, 2001a. p. 401-420.

Auto-implante esplênico. In: (Ed.). Terapêutica cirúrgica: indicações, decisões, tática, técnica. Rio de Janeiro: Guanabara Koogan, 2001b. p. 421-427.

PISTER, P. W. T.; PACHTER, H. L. Autologous splenic transplantation. Ann. Surg., Madison, v. 219, n. 3, p. $225-$ $235,1994$.

RESENDE, V.; PETROIANU, A. Funções do remanescente esplênico após esplenectomia subtotal para o tratamento de lesões complexas do baço humano. Revta Assoc. Med. Bras., São Paulo, v. 48, n. 1, p. 26-31, 2002.

RODRIGUES, A. Z.; NIGRO, A. J. T.; PAIVA, E. R.; SEMENTILLI, A.; TAVARES, N. M. Estudo comparativo da viabilidade de fragmento de tecido esplênico autólogo implantado no omento maior ou na tela subcutânea em ratas. Acta Cir. Bras., Rio de Janeiro, v. 6, n. 2, p. 64-67. 1991.

SCHALLER, O. Nomenclatura anatômica veterinária ilustrada. São Paulo: Manole, 1999. 306 p.

SCHMIDT, S.; LOHSE, C. L.; SUTER, P. F. Branching Pat-Terns of the hepatic artery in the dog: arteriographic and anatomic study. Am. J. Vet. Res., Chicago, v. 41, n. 7, p. 1090-1097, 1980.

SIMÕES, F. C.; MARQUES, R. G.; DIESTEL, C. F.; CAETANO, C. E. R.; DINIS, A. P. G.; HORST, N. L.; NETO, J. F. N.; PORTELA, M. C. 2007. Lipidic profile among rats submitted to total splenectomy isolated or combined with splenic autotransplant. Acta Cir. Bras., Rio de Janeiro, v. 22, n. 1, p. 46-51, 2007.

SOUZA, J. C. L.; ATHIÊ, E.; MARIGO, C.; RAHAL, F.; FAGUNDES, D. J. Estudo da regeneração esplênica autóloga e heterotópica em ratos. Acta Cir. Bras., Rio de Janeiro, v. 20, n. 3, p. 253-257, 2005.

XAVIER-SILVA, B.; ROZA, M. S.; HERNANDEZ, J. M. F.; SOUZA, H. J. M de; ABIDU-FIGUEIREDO, M. Artéria lienal em gatos: estudo aplicado à pesquisa anatômica e a prática cirúrgica. Revista Brasileira de Medicina Veterinária, Rio de Janeiro, v. 33, n. 1, p. 41-47, 2011.

ZÚNIGA, C. T.; MOLINA, H. Z.; ALVAREZ, R. U.; SEGUEL, E. S.; BENAVIDES, C. Y.; AROSTEGUI, C. P.; ARANCIBIA, H. Z.; AMSTEIN, L. P. Traumatismo esplénico, experiencia en el manejo quirúrgico. Revta Chil. Cir., Chile, v. 54, n. 1, p. 79-84, 2002. 
